\title{
On algebraic curves for commuting elements in $q$-Heisenberg algebras
}

\author{
Johan RICHTER and Sergei SILVESTROV \\ Centre for Mathematical Sciences, Lund University, P.O. Box 118, \\ SE-221 00 Lund, Sweden \\ E-mails: johanr@maths.lth.se,ssilvest@maths.lth.se
}

\begin{abstract}
In the present article we continue investigating the algebraic dependence of commuting elements in $q$-deformed Heisenberg algebras. We provide a simple proof that the 0 -chain subalgebra is a maximal commutative subalgebra when $q$ is of free type and that it coincides with the centralizer (commutant) of any one of its elements different from the scalar multiples of the unity. We review the Burchnall-Chaundy-type construction for proving algebraic dependence and obtaining corresponding algebraic curves for commuting elements in the $q$-deformed Heisenberg algebra by computing a certain determinant with entries depending on two commuting variables and one of the generators. The coefficients in front of the powers of the generator in the expansion of the determinant are polynomials in the two variables defining some algebraic curves and annihilating the two commuting elements. We show that for the elements from the 0-chain subalgebra exactly one algebraic curve arises in the expansion of the determinant. Finally, we present several examples of computation of such algebraic curves and also make some observations on the properties of these curves.
\end{abstract}

2000 MSC: 16S99, 81S05, 39A13

\section{Introduction}

In 1994, one of the authors of the present paper, S. Silvestrov, based on consideration of the previous literature and a series of trial computations, made the following three-part conjecture.

- The first part of the conjecture stated that the Burchnall-Chaundy-type result on algebraic dependence of commuting elements can be proved in greater generality, that is, for much more general classes of noncommutative algebras and rings than the Heisenberg algebra and related algebras of differential operators treated by Burchnall and Chaundy and in subsequent literature [1, 2, 3, 7, 8, 11].

- The second part stated that the Burchnall-Chaundy eliminant construction of annihilating algebraic curves formulated in determinant (resultant) form works after some appropriate modifications for most or possibly all classes of algebras where the BurchnallChaundy-type result on algebraic dependence of commuting elements can be proved.

- Finally, the third part of the conjecture stated that the proof of the vanishing of the corresponding determinant algebraic curves on the commuting elements can be performed in a purely algebraic way for all classes of algebras or rings where this fact is true, that is, using only the internal structure and calculations with the elements in the corresponding algebras or rings and the algebraic combinatorial expansion formulas for the corresponding determinants without any need of passing to operator representations and use of analytic methods as in the Burchnall-Chaundy-type proofs. 
This third part of the conjecture remains widely open with no general such proofs available for any classes of algebras and rings, even in the case of the usual Heisenberg algebra and differential operators, and with only a series of examples calculated for the Heisenberg algebra, $q$-Heisenberg algebra, and some more general algebras, all supporting the conjecture. In the first and second parts of the conjecture progress has been made. In [4], the key Burchnall-Chaundy-type theorem on algebraic dependence of commuting elements in $q$-deformed Heisenberg algebras (and thus as a corollary for $q$-difference operators as operators representing $q$-deformed Heisenberg algebras) was obtained. The result and the methods have been extended to more general algebras and rings generalizing $q$-deformed Heisenberg algebras (generalized Weyl structures and graded rings) in [5]. The proof in [4] is totally different from the Burchnall-Chaundy-type proof. It is an existence argument based only on the intrinsic properties of the elements and internal structure of $q$-deformed Heisenberg algebras, thus supporting the first part of the conjecture. It can be used successfully for an algorithmic implementation for computing the corresponding algebraic curves for given commuting elements. However, it does not give any specific information on the structure or properties of such algebraic curves or any general formulae. It is thus important to have a way of describing such algebraic curves by some explicit formulae, as, for example, those obtained using the Burchnall-Chaundy eliminant construction for the $q=1$ case, that is, for the classical Heisenberg algebra. In [10], a step in that direction was taken by offering a number of examples, all supporting the claim that the eliminant determinant method should work in the general case. However, no general proof for this was provided. The complete proof following the Burchnall-Chaundy approach in the case of $q$ not a root of unity has been recently obtained [6], by showing that the determinant eliminant construction, properly adjusted for the $q$-deformed Heisenberg algebras, gives annihilating curves for commuting elements in the $q$-deformed Heisenberg algebra when $q$ is not a root of unity, thus confirming the second part of the conjecture for these algebras. That proof was obtained by adapting the Burchnall-Chaundy eliminant determinant method of the case $q=1$ of differential operators to the $q$-deformed case, after passing to a specific faithful representation of the $q$-deformed Heisenberg algebra on Laurent series and then performing a detailed analysis of the kernels of arbitrary operators in the image of this representation. While exploring the determinant eliminant construction of the annihilating curves, we also obtained some further information on such curves and some other results on dimensions and bases in the eigenspaces of the $q$ difference operators in the image of the chosen representation of the $q$-deformed Heisenberg algebra. Recently, a further extension of Burchnall-Chaundy eliminant determinant method to the context of $\sigma$-derivations and Ore extension rings has been considered in [9]. In the case of $q$ being a root of unity the algebraic dependence of commuting elements holds only over the center of the $q$-deformed Heisenberg algebra [4], and it is still unknown how to modify the eliminant determinant construction to yield annihilating curves for this case.

In the present article we continue investigation of the algebraic dependence of commuting elements in $q$-deformed Heisenberg algebras within the context of $[4,6,10]$. In Section 2, following [4], we recall some preliminaries on $q$-deformed Heisenberg algebra, including degree function, decomposition into the direct sum of the "chain" subspaces indexed by the integers and corresponding to this decomposition the upper and lower chain functions. In Section 3, we consider in more detail the 0-chain subspace (indexed by zero). This subspace is a commutative subalgebra in the $q$-deformed Heisenberg algebra playing a pivotal role for the structure of this algebra [4]. We provide a simple proof that this subalgebra is a maximal commutative subalgebra when $q$ is of free type, and that it coincides with the centralizer (commutant) of any one of its elements different from the scalar multiples of the 
unity. In Section 4, we review the Burchnall-Chaundy-type construction for proving algebraic dependence and obtaining corresponding algebraic curves for commuting elements in the $q$ deformed Heisenberg algebra following [6] but putting it into general context of the elements of the $q$-deformed Heisenberg algebra rather then operators of a specific representation. The construction is based on computing a certain determinant of a matrix with entries depending on two commuting variables and containing one of the generators of the $q$-deformed Heisenberg algebra. This matrix is constructed from commuting elements. The coefficients in front of the powers of the generator in the expansion of the determinant are polynomials in the two variables defining some algebraic curves. The commuting elements satisfy the equations of these algebraic curves [6]. In Section 5, we show that for the elements from the 0-chain subalgebra exactly one algebraic curve arises via this construction in the expansion of the determinant and then present several examples of computations of such algebraic curves and also make some observations on the properties of these curves based on these examples and further computer experiments.

\section{Preliminaries}

Let $K$ be a field of characteristic 0 , and $q$ a nonzero element of $K$. We say that $q$ is of free type if it is 1 or not a root of unity. If $q$ is a root of unity, we say it is of torsion type. We define the $q$-deformed Heisenberg algebra over $K$ as

$$
H(q)=K\langle A, B\rangle /(A B-q B A-I)
$$

The identity element will be denoted by $I$. For $q=1$ we recover the classical Heisenberg algebra (called also Weyl algebra). One can define degree functions $\operatorname{deg}_{A}$ and $\operatorname{deg}_{B}$ with respect to $A$ and $B$ on $H(q)$ just as on the commutative algebra of polynomials. One computes these functions by inspection just as one would in a commutative algebra. That the functions are well defined and does not depend on how the elements are written is proved in $[4$, Chapter 4]. We also define the total degree function $\operatorname{deg}(\alpha)=\operatorname{deg}_{A}(\alpha)+\operatorname{deg}_{B}(\alpha)$. In $[4$, Chapter 4] the following theorem is proved.

Theorem 2.1. Let $\alpha, \beta \in H(q)$ for some $q \neq 0$ and let $V \in\{A, B\}$. Then

$$
\operatorname{deg}_{V}(\alpha \beta)=\operatorname{deg}_{V}(\alpha)+\operatorname{deg}_{V}(\beta)
$$

We define the sets $R_{n}$ for all integers $n$ by

$$
R_{n}=\left\{\sum_{\substack{j \geq 0 \\ j+n \geq 0}} a_{j} B^{j+n} A^{j} \mid a_{j} \in K, a_{j} \neq 0 \text { for at most finitely many } j\right\}
$$

If the element $\alpha \in H(q)$ belongs to some $R_{n}$, we say that it is homogeneous. We also define a function

$$
\chi:\{\alpha \in H(q) \mid \alpha \text { is nonzero and homogeneous }\} \longrightarrow \mathbb{Z}
$$

by defining $\chi(\alpha)$ to be the unique integer such that $\alpha \in R_{\chi(\alpha)}$. This function is called the chain function.

All $R_{i}$ are vector spaces over $K$. Further $H(q)$ is the direct sum of all the $R_{i}$. We can use this to define a projection operation. Let $\alpha$ be an element of $H(q)$. We can write $\alpha=\sum_{j} \alpha_{j}$, 
where $\alpha_{j} \in R_{j}$. This decomposition is unique. We then define the projection of $\alpha$ on $R_{n}$ by $\alpha \sqcap R_{n}=\alpha_{n}$. The notation is intended to recall the notation for intersection. At this point we define two new functions. They are defined for all nonzero elements of $H(q)$.

$$
\bar{\chi}(\alpha)=\max \left\{n \in \mathbb{Z} \mid \alpha \sqcap R_{n} \neq 0\right\}, \quad \underline{\chi}(\alpha)=\min \left\{n \in \mathbb{Z} \mid \alpha \sqcap R_{n} \neq 0\right\}
$$

These functions are known as the upper and lower chain functions, respectively.

\section{$3 \quad R_{0}$ is maximal commutative}

We begin by noting that all elements of $R_{0}$ commute with each other [4]. Furthermore, the products of two elements $\alpha, \beta \in R_{0}$ are in $R_{0}$. So $R_{0}$ is a commutative subalgebra. We want to show that it is in fact a maximal commutative subalgebra.

For an element $\alpha \in H(q)$ we define $\operatorname{Cen}(\alpha)=\{\beta \in H(q) \mid[\alpha, \beta]=0\}$.

In $[4$, Chapter 6$]$ the following theorem is proved (as a part of Theorem 6.10).

Theorem 3.1. Let $q$ be of free type. Let $\alpha, \beta$ be two commuting elements in $H(q)$. Then the following is true:

- If $\bar{\chi}(\alpha)=0$, then $\bar{\chi}(\beta)=0$ or $\alpha \sqcap R_{0}=c I$ for some $c \in K$.

- If $\underline{\chi}(\alpha)=0$, then $\underline{\chi}(\beta)=0$ or $\alpha \sqcap R_{0}=c I$ for some $c \in K$.

We now describe the centralizer of an element in $R_{0}$.

Theorem 3.2. Let $q$ be of free type and $\alpha \in R_{0} \subset H(q)$. Assume further that $\alpha \neq c I$ for all $c \in K$. Then $\operatorname{Cen}(\alpha)=R_{0}$.

Proof. As we noted above $R_{0} \subseteq C e n(\alpha)$. It remains to show the other inclusion. Let $\beta$ be an arbitrary nonzero element of $\operatorname{Cen}(\alpha)$. By Theorem 3.1 we must have $\bar{\chi}(\beta)=0$, since $\alpha \sqcap R_{0} \neq c I$. Similarly we must have that $\underline{\chi}(\beta)=0$. So in the direct sum decomposition only elements in $R_{0}$ occurs. Thus $\beta \in R_{0}$.

Corollary 3.3. $R_{0}$ is maximal commutative.

Proof. Let $\beta$ be an element that commutes with everything in $R_{0}$. Then in particular it must commute with $B A$. But $C e n(B A)=R_{0}$ by the preceding theorem. Thus $\beta \in R_{0}$.

\section{Annihilating polynomials}

As mentioned in the introduction any two commuting elements in $H(q)$ must be algebraically dependent when $q$ is of free type. More formally, we have the following.

Theorem 4.1. Let $q \in K$ be of free type. If $\alpha, \beta \in H(q)$ commute, then there exists a nonzero $P \in K[x, y]$ such that $P(\alpha, \beta)=0$.

We now describe an explicit construction of this polynomial. We let $s$ and $t$ be variables that take values in the base field $K$. We write the commuting elements $\alpha, \beta$ as

$$
\alpha=\sum_{i=0}^{n} p_{i}(B) A^{i}, \quad \beta=\sum_{i=0}^{m} r_{i}(B) A^{i}
$$

where the $p_{i}$ and $r_{i}$ are polynomials. We will form an $n+m$ determinant that will give us the annihilating polynomial. 
Consider the expressions obtained by reordering all $A$ to the right of $B$ in

$$
\begin{aligned}
A^{k}(\alpha-s I) & =\sum_{i=0}^{n+k} \theta_{i, k} A^{i}, \quad k=0,1, \ldots, m-1 \\
A^{k}(\beta-t I) & =\sum_{i=0}^{m+k} \omega_{i, k} A^{i}, \quad k=0,1, \ldots, n-1
\end{aligned}
$$

where $\theta_{i, k}$ and $\omega_{i, k}$ are functions of $B, s, t$ arising after reordering. The coefficients of the powers of $A$ will be the elements in the determinant that we compute. $\theta_{i, k}$ will be placed as the element in row $k+1$ and column $i . \omega_{i, k}$ will be placed in row $k+m+1$ and column $i$. The determinant will thus be a polynomial in $s, t$ and $B$. This polynomial, which we will call the eliminant of $\alpha$ and $\beta$, can be written as $\sum_{i} \delta_{i}(s, t) B^{i}$. Every such $\delta_{i}$ will satisfy $\delta_{i}(\alpha, \beta)=0$ and at least one of them will not be identically zero.

A more precise formulation with additional information about the construction can be found in the following.

Theorem 4.2. Let

$$
\alpha=\sum_{j=0}^{m} p_{j}(B) A^{j}, \quad \beta=\sum_{j=0}^{n} r_{j}(B) A^{j}
$$

be two commuting elements, the $p_{j}$ and $r_{j}$ being polynomials, and denote their eliminant by $\Delta_{\alpha, \beta}(B, s, t)$. Then $\Delta_{\alpha, \beta} \neq 0$. Furthermore $\Delta_{\alpha, \beta}$ has degree $n$ seen as polynomial in $s$. If $r_{n}(B)=\sum_{i} a_{i} B^{i}\left(a_{i} \in K\right)$, then $\Delta_{\alpha, \beta}$ has leading coefficient $(-1)^{n} \prod_{k=0}^{m-1}\left(\sum_{i} a_{i} q^{k i} B^{i}\right)$, once again seen as a polynomial in s. Symmetrically, $\Delta_{\alpha, \beta}$ will have degree $m$ seen as a polynomial in $t$. The coefficient of $t^{m}$ will be $(-1)^{m} \prod_{k=0}^{n-1}\left(\sum_{i} b_{i} q^{k i} B^{i}\right)$ if $p_{m}(B)=\sum_{i} b_{i} B^{i}$.

Let $g=n \max _{j} \operatorname{deg}\left(p_{j}\right)+m \max _{j} \operatorname{deg}\left(r_{j}\right)$. We can write

$$
\Delta_{\alpha, \beta}(B, s, t)=\sum_{i=0}^{g} \delta_{i}(s, t) B^{i}
$$

Then at least one $\delta_{i}(s, t) \neq 0$ and $\delta_{i}(\alpha, \beta)=0$ for all $i$.

\section{The eliminant when the elements belong to $R_{0}$}

In the general case the theorem does not rule out that one can get several nonzero $\delta_{i}$ in the expansion of the eliminant, $\Delta_{\alpha, \beta}$. This does not, however, occur when $\alpha$ and $\beta$ belong to $R_{0}$.

Theorem 5.1. Let $\alpha=\sum_{k=0}^{n} p_{k} B^{k} A^{k}$ and $\beta=\sum_{l=0}^{m} r_{k} B^{l} A^{l}$. Then, with the same notation as before, there will be only one nonzero $\delta_{i}$ when the eliminant is computed and this $i$ will equal $\mathrm{nm}$.

Proof. We begin by noting that $A^{n}(\alpha-s I)$ will be of the form $\sum_{i=n}^{n+k} a_{i} B^{i-k} A^{i}$, where the $a_{i}$ belong to $K$.

We use this result to describe the structure of the eliminant. Denote the element in row $u$ and column $v$ by $e_{u, v}$. Then we will have $e_{u, v}=\phi_{i, j}(s, t) B^{v-u}$ if $u \leq m$ (that is in the first $\mathrm{m}$ rows) and $e_{u, v}=\phi_{i, j} B^{v-u+m}$ otherwise (in the last $\mathrm{n}$ rows), where the $\phi_{i, j}(s, t)$ are polynomials over $K$. Many of them will of course be zero, in particular those where $B$ would otherwise occur with a negative exponent. 
We know, from ordinary linear algebra, that

$$
\Delta_{\alpha, \beta}(B, s, t)=\sum_{\sigma} \operatorname{sign}(\sigma) \prod_{g=1}^{m+n} e_{g, \sigma(g)}
$$

where $\sigma$ denotes a permutation. But looking at an arbitrary term of the sum we find that it can be written as

$$
\begin{aligned}
\operatorname{sign}(\sigma) & \prod_{g=1}^{g=m}\left(\phi_{g, \sigma(g)}(s, t) B^{\sigma(g)-g}\right) * \prod_{g=m+1}^{m+n} \phi_{g, \sigma(g)}(s, t) B^{\sigma(g)-g+m} \\
= & \Phi(s, t) * B^{\sum_{g=1}^{m+n}(\sigma(g))-\sum_{g=1}^{n+m}(g)+m n}
\end{aligned}
$$

for a polynomial $\Phi(s, t)$. But the two sums in the exponent cancel, since they have the same terms in different order, and we conclude that we get the exponent $m n$. Since we picked an arbitrary term, we are done.

\subsection{Examples}

We will include some examples here to give a feeling for the construction of the eliminant and our result. Let $\alpha=B A$ and $\beta=B^{2} A^{2}$. Then

$$
\Delta_{\alpha, \beta}(B, s, t)=\left|\begin{array}{ccc}
-s & B & 0 \\
0 & 1-s & q B \\
-t & 0 & B^{2}
\end{array}\right|
$$

On computing the determinant we find that the annihilating polynomial is $s^{2}-s-t q$. This is only a slight modification of the classical case when $q=1$. (We note that it makes no difference whether we set $q=1$ at the beginning of the calculation or the end.)

For our next example let $\alpha$ be as before and let $\beta=B^{3} A^{3}$. Then we find that

$$
\Delta_{\alpha, \beta}(B, s, t)=\left|\begin{array}{cccc}
-s & B & 0 & 0 \\
0 & 1-s & q B & 0 \\
0 & 0 & 1+q-s & q^{2} B \\
-t & 0 & 0 & B^{3}
\end{array}\right|
$$

We get the annihilating polynomial

$$
s^{3}-(2+q) s^{2}+(1+q) s-q^{3} t
$$

Once again no essential simplification occurs if we let $q$ approach 1 .

Now set $\beta=B^{2} A^{2}$ and $\alpha=B^{3} A^{3}$. The determinant becomes

$$
\Delta_{\alpha, \beta}(B, s, t)=\left|\begin{array}{ccccc}
-s & 0 & 0 & B^{3} & 0 \\
0 & -s & 0 & \left(1+q+q^{2}\right) B^{2} & q^{3} B^{3} \\
-t & 0 & B^{2} & 0 & 0 \\
0 & -t & (1+q) B & q^{2} B^{2} & 0 \\
0 & 0 & 1+q-t & q\left(1+2 q+q^{2}\right) B & q^{4} B^{2}
\end{array}\right|
$$


and we get the annihilating polynomial

$$
q^{3} s^{2}+\left(q+2 q^{2}\right) s t+(1+q) t^{2}-t^{3}
$$

In the classical case this polynomial becomes

$$
s^{2}+3 s t+2 t^{2}-t^{3}
$$

As a final example we can take $\alpha=B^{2} A^{2}$ and $\beta=B^{4} A^{4}$. The eliminant is

$$
\Delta_{\alpha, \beta}(B, s, t)=\left|\begin{array}{cccccc}
-s & 0 & B^{2} & 0 & 0 & 0 \\
0 & -s & (1+q) B & q^{2} B^{2} & 0 & 0 \\
0 & 0 & 1+q-s & q\left(1+2 q+q^{2}\right) B & q^{4} B^{2} & 0 \\
0 & 0 & 0 & 1+2 q+2 q^{2}+q^{3}-s & q^{2}\left(1+2 q+2 q^{2}+q^{3}\right) B & q^{6} B^{2} \\
-t & 0 & 0 & 0 & B^{4} & 0 \\
0 & -t & 0 & 0 & \left(1+q+q^{2}+q^{3}\right) B^{3} & q^{4} B^{4}
\end{array}\right|
$$

We then get the annihilating polynomial

$$
\begin{aligned}
q^{8} t^{2} & -2 q^{6} t s-3 q^{5} t s-2 q^{4} t s^{2}-2 q^{4} t s-q^{3} t s+q^{4} s^{2}-q^{3} s^{3}+3 q^{3} s^{2} \\
& -2 q^{2} s^{3}+4 q^{2} s^{2}-3 q s^{3}+3 q s^{2}+s^{2}+s^{4}-2 s^{3}
\end{aligned}
$$

The limit when $q$ goes towards 1 is

$$
t^{2}-8 t s-2 s^{2} t+12 s^{2}-8 s^{3}+s^{4}
$$

This is a simpler expression but only because the coefficients are simpler. No coefficient has become zero.

This illustrates that the complexity of the resulting polynomial grows pretty fast.

Computer experiments indicate that Theorem 5.1 can be generalized substantially. We would be also interested to know whether the annihilating polynomials always have genus 0 , a conjecture we have been unable to find any counterexamples to.

\section{Acknowledgements}

This work was supported by the Swedish Research Council, the Swedish Foundation for International Cooperation in Research and Higher Education (STINT), the Crafoord Foundation, the Royal Physiographic Society in Lund, and the Royal Swedish Academy of Sciences.

\section{References}

[1] J. L. Burchnall and T. W. Chaundy. Commutative ordinary differential operators. Proc. Lond. Math. Soc. (2), 21 (1922), 420-440.

[2] J. L. Burchnall and T. W. Chaundy. Commutative ordinary differential operators. Proc. R. Soc. Lond. Ser. A Math. Phys. Eng. Sci., 118 (1928), 557-583.

[3] J. L. Burchnall and T. W. Chaundy. Commutative ordinary differential operators. II. The Identity $P^{n}=Q^{m}$. Proc. R. Soc. Lond. Ser. A Math. Phys. Eng. Sci., 134 (1932), 471-485.

[4] L. Hellström and S. Silvestrov. Commuting Elements in q-Deformed Heisenberg Algebras. World Scientific Publishing, River Edge, NJ, 2000. 
[5] L. Hellström and S. Silvestrov. Ergodipotent maps and commutativity of elements in noncommutative rings and algebras with twisted intertwining. J. Algebra, 314 (2007), 17-41.

[6] M. de Jeu, C. Svensson, and S. Silvestrov. Algebraic curves for commuting elements in the q-deformed Heisenberg algebra. J. Algebra, 321 (2009), 1239-1255.

[7] I. Krichever. Integration of nonlinear equations by the methods of algebraic geometry (Russian). Funktsional. Anal. i Priložen, 11 (1977), 15-31.

[8] I. Krichever. Methods of algebraic geometry in the theory of nonlinear equations (Russian). Uspekhi Mat. Nauk, 32 (1977), 183-208.

[9] D. Larsson. Burchnall-Chaundy theory, Ore extensions and $\sigma$-differential operators. U.U.D.M. Report 2008:45, Department of Mathematics, Uppsala University, 2008.

[10] D. Larsson and S. D. Silvestrov. Burchnall-Chaundy theory for $q$-difference operators and $q$ deformed Heisenberg algebras. J. Nonlinear Math. Phys., 10 (2003), Suppl. 2, 95-106.

[11] D. Mumford. An algebro-geometric construction of commuting operators and of solutions to the Toda lattice equation, Korteweg deVries equation and related nonlinear equation. Proceedings of the International Symposium on Algebraic Geometry (Kyoto University, Kyoto, 1977), 1978, $115-153$.

Received May 21, 2009

Revised September 20, 2009 